University of Nebraska - Lincoln

DigitalCommons@University of Nebraska - Lincoln

1999

\title{
Thirteen-lined Ground Squirrel (Sciuridae: Spermophilus tridecemlineatus) Antipredator Vigilance: Monitoring the Sky for Aerial Predators
}

\author{
Cody L. Arenz \\ University of Nebraska-Lincoln, darenz@conpoint.com \\ Daniel Leger \\ University of Nebraska-Lincoln, dleger1@unl.edu
}

Follow this and additional works at: https://digitalcommons.unl.edu/psychfacpub

Part of the Psychiatry and Psychology Commons

Arenz, Cody L. and Leger, Daniel, "Thirteen-lined Ground Squirrel (Sciuridae: Spermophilus tridecemlineatus) Antipredator Vigilance: Monitoring the Sky for Aerial Predators" (1999). Faculty Publications, Department of Psychology. 537.

https://digitalcommons.unl.edu/psychfacpub/537

This Article is brought to you for free and open access by the Psychology, Department of at DigitalCommons@University of Nebraska - Lincoln. It has been accepted for inclusion in Faculty Publications, Department of Psychology by an authorized administrator of DigitalCommons@University of Nebraska - Lincoln. 
Published in Ethology 105:9 (1999), pp. 807-816; doi: 10.1046/j.1439-0310.1999.00458.x

Copyright (c) 1999 Blackwell Wissenschafts-Verlag, Berlin. Used by permission.

Submitted October 10, 1998; accepted February 8, 1999.

\title{
Thirteen-lined Ground Squirrel (Sciuridae: Spermophilus tridecemlineatus) Antipredator Vigilance: Monitoring the Sky for Aerial Predators
}

\author{
Cody L. Arenz \& Daniel W. Leger \\ Nebraska Behavioral Biology Group, School of Biological Sciences and \\ Department of Psychology, University of Nebraska-Lincoln \\ Corresponding author - Cody L. Arenz, 203 S 9th St, Norfolk, NE 68701, USA; \\ email darenz@conpoint.com
}

\begin{abstract}
Despite the success of antipredator vigilance research, the specific focus of this vigilance has been difficult to determine. We have previously shown that thirteen-lined ground squirrels (Spermophilus tridecemlineatus) increase their vigilance when their lateral field of view is obstructed. In this paper, we describe an experiment in which we attempt to determine the predator class for which this vigilance is directed. Using six differentially occluded Plexiglas foraging boxes with hinged "eaves," we were able to obstruct the squirrels' view of the sky while not obstructing their view of terrestrial threats. In general, across the box types, when their sky view was obstructed, ground squirrels increased their vigilance by increasing the percentage of time spent withdrawn from the boxes. This result suggests that a significant portion of ground squirrel antipredator vigilance is directed at the sky and is surveillance for aerial predators.
\end{abstract}

\section{Introduction}

The importance of predation in influencing behavioral repertoires, life histories, and species interactions may seem obvious, but fine-grained empirical assessment of what affects an animal's perception of its predation risk has only recently been an area of focus (Lima 1998). In a literal sense, we are unable to determine what an animal perceives and instead rely on externally observable actions that indicate what an animal perceives as high risk. In this context, the most 
commonly observed behavior is antipredator vigilance. Simply, this is merely visual scanning of the animal's surroundings (Lazarus 1990), an increase in which increases the likelihood that a stimulus will be detected (Dimond \& Lazarus 1974). Vigilance is one of the most commonly reported antipredator behaviors (Elgar 1989; Lima \& Dill 1990; Quenette 1990). Many variables have been shown to be responsible for the observed variation in vigilance, including group size, temperature, time of day, predator presence, distance from refuge, sex (Elgar 1989; Quenette 1990), group geometry (Bekoff 1995; Sadedin \& Elgar 1998), visual obstruction (Metcalfe 1984; Lima 1991, 1992; Lazarus \& Symonds 1992; Arenz \& Leger 1997a, b;), nutritional need or foraging pressure (Bachman 1993), and the utility and cost of vigilance (Scheel 1993; Arenz \& Leger 1999).

Experimental tests have shown that animals may increase their vigilance when visual obstructions are present (Lima 1991, 1992). Further tests suggest that whether the visual obstruction can also serve as protective cover is important (Lazarus \& Symonds 1992). However, for some species, merely the amount of visual field obstructed is not likely to be the only important factor (Arenz \& Leger 1997a). Instead, the position of visual obstruction within the animal's visual field can be a significant influence upon vigilance. Arenz \& Leger (1997a) have shown that thirteen-lined ground squirrels (Spermophilus tridecemlineatus), despite having a visual field of 360 degrees, greatly increase their vigilance only when their lateral field of view is obstructed. This increase in vigilance is primarily due to the obstruction of their long-range lateral view (Arenz \& Leger 1997b); obstruction of the overhead and frontal fields of view did not result in increased vigilance.

The general conclusion that can be drawn from these visual obstruction studies, and from antipredator vigilance research as a whole, is that antipredator vigilance increases as predation risk increases (Roberts 1996). Although it does not affect our ability to assign an antipredator function to vigilance, one frustrating aspect of studying this behavior is that it is difficult to determine the specific focus of an animal's visual scans. Some authors have inferred this information from gaze direction (Caine \& Marra 1988), but usually we are unable to know whether a prey animal is more concerned with, for example, attack from below or attack from the side. In this study, we have initiated the process of determining the classes of predators for which thirteen-lined ground squirrels (Spermophilus tridecemlineatus) are vigilant. That is, the research question dealt with here is whether a significant portion of ground squirrel vigilance is directed toward aerial predators. In this study, we have used Plexiglas foraging boxes that obstruct the squirrels' view of the sky. The hypothesis being tested is that ground squirrels increase their antipredator vigilance if their "sky-view" is obstructed.

In summary, previous research has shown that the presence of visual obstruction results in an increase in vigilance. Therefore, this visual obstruction obviously prevents the animal from obtaining certain important information. Yet we have previously been unable to determine the focus of antipredator vigilance (Roberts 1996). The following experiment has been designed to determine whether aerial predators are a significant focus of thirteen-lined ground squirrel antipredator vigilance. 


\section{Methods}

\section{Study Site and Subjects}

Thirteen-lined ground squirrels are small (100-200 g, 20-30 cm in total length), burrowing, grassland rodents that occur across the Great Plains of North America.

Current suitable habitat often exists as human-managed areas such as cemeteries and parks (Jones et al. 1985), but they are sometimes found in pastures and roadside grassy areas as well (Arenz, pers. obs.). Although these ground squirrels are relatively asocial (Vestal \& McCarley 1984), the presence of high-quality habitat can lead to densities of $25 /$ ha or more (McCarley 1966). Home ranges tend to be large (about 1-4.5 ha) and overlapping (McCarley 1966; Vestal \& McCarley 1984). A wide variety of predators include ground squirrels in their diet, including snakes (e.g. bullsnake, Pituophis melanoleucus), raptors (e.g. red-tailed hawk, Buteo jamaicensis), canids (e.g. red fox, Vulpes vulpes), and felids (domestic cat, Felis domesticus). Representatives of all of these predator groups (except snakes) were observed at the study site. Red-tailed hawks have been observed capturing squirrels at the study site.

This work was carried out in Wyuka Cemetery in Lincoln, Nebraska, USA. Behavioral observations for this and another experiment (Arenz \& Leger 1999) occurred from March 1 to July 26, 1997, and the testing of subjects for this experiment occurred from June 4 to July 26, 1997. This testing period occurred during the time of pup emergence (June 16-18, 1997), but avoided the mating period (ca. fourth wk of Mar. 1997). The cemetery is approximately 60.7 ha in area, but all of the observations occurred on approximately 5.3-ha, which encompassed parts or all of the home ranges of about 35 adult ground squirrels (over the entire season). The study site was mown regularly (ca. every $5 \mathrm{~d}$ ) to a height of $4-5 \mathrm{~cm}$. The visual obstruction of the ground squirrel habitat in the cemetery varied with tombstone type (flush with the ground or erect), tree density, and the presence or absence of graves. The 5.3-ha study site contained flush stones, several large trees, and a scattering of small flower vases near the stones (ca. 16-28 cm in height).

Ground squirrel adults (21 females, 6 males) were live-trapped at burrow entrances with Tomahawk ${ }^{\circledR}$ live traps. Not all of these individuals remained throughout testing and were presumed to have dispersed or died. Ground squirrels were restrained using a canvas bag (Arenz 1997), uniquely dye-marked with nyanzol-A dye (Melchior \& Iwen 1965), ear-tagged with a fingerling tag, and sexed before being released. Two males and 6 females were presented with all 6 experimental conditions. These squirrels were subjects in another experiment (Arenz \& Leger 1999) that overlapped in time with this one. However, each squirrel completed the other experiment before becoming part of this study.

Observation of ground squirrel movements to estimate home range sizes occurred intensively and daily from April 10 to May 19, 1997, with additional observations taking place later in the season (June 5 to June 26, 1997). Home range size was estimated and reevaluated during the season to ensure that the test site for each squirrel (see below) was near the center of that squirrel's home range (it was not necessary to move test sites for any subject). We chose test sites near 
the center of home ranges to reduce variation unassociated with the experimental conditions and to reduce trial interruptions from other ground squirrels that might occur if the test sites happened to be on the edges of home ranges.

\section{Apparatus}

Six boxes $(10.2 \times 10.2 \times 15.2 \mathrm{~cm})$ were constructed from 3.2-mm clear Plexiglas (Table 1). These boxes were clear on the sides and end, and Plexiglas eaves $(10.2 \times 15.2 \mathrm{~cm})$ were attached to them using clear plastic hinges along the top edge of the box.

The word "eave" is an architectural term describing a projecting overhang from the lower portion of a roof. We used eaves to obstruct the foraging squirrels' view of the sky, but not their view of the potential lateral approach of predators. Since the eaves could be rotated with the hinges, we were able to adjust for minor topographical variation that occurred between the testing locations such that only the sky was obstructed. We used three box configurations with one box of each pair having clear eaves and one having opaque eaves. The first pair (C1 and O1) had two eaves, one on each side; and the top of the box was clear. The second pair (C2 and O2) also had two eaves, but the top of the box was opaque. Therefore, the only difference between the first and second pair of boxes is whether the top of the box was clear or opaque. Arenz \& Leger (1997a) found that there were no significant differences in the antipredator vigilance of adult thirteen-lined ground squirrels to a clear box and a box that was occluded on the top and end. Therefore, it would appear that the portion of their field of view that was directly overhead is relatively unimportant. However, in the current experiment, all boxes had clear ends and therefore are not the same as the boxes in Arenz \& Leger (1997a). The inclusion of C1 and O1 was to determine whether our original finding, that the overhead view was unimportant, could be supported in this experiment. The third pair of boxes (C3 and O3) had a third eave $(10.2 \times 15.2 \mathrm{~cm})$ over the end of the box. Therefore, the third pair of boxes either controlled for (C3) or obstructed (O3) the ground squirrels' lateral and anterior view of the sky. The first two pairs of boxes either controlled for $(\mathrm{C} 1, \mathrm{C} 2)$ or obstructed $(\mathrm{O} 1, \mathrm{O} 2)$ the ground squirrels' lateral view of the sky. We

Table 1. Description of the six Plexiglas boxes used to manipulate the ground squirrels' view of the sky

\begin{tabular}{llll}
\hline Box type symbol & Top of box & No. of eaves & Type of eaves \\
\hline C1 & clear & 2 & on the sides, clear \\
O1 & clear & 2 & on the sides, opaque \\
C2 & opaque & 2 & on the sides, clear \\
O2 & opaque & 2 & on the sides, opaque \\
C3 & opaque & 3 & on the sides and end, clear \\
O3 & opaque & 3 & on the sides and end, opaque \\
\hline
\end{tabular}


expected that further obstruction of the squirrels' sky-view would be perceived as further increasing predation risk. At the time of presentation, each box contained about 8.5-g of peanut butter, a highly preferred food item that had to be consumed in the box.

\section{Procedure}

We assigned box treatment order through a randomized block design with squirrels as the blocking factor. Each squirrel was tested with each box type at least once (total number of scheduled trials $=48$ ). Some repetition occurred because these squirrels were free-ranging and their home ranges overlapped considerably; therefore, sometimes a nontargeted squirrel entered the Plexiglas box. When this occurred, rather than either frightening the squirrel away or wasting potential data, the nontargeted squirrel's behavioral measures were averaged with those obtained from its scheduled presentation of that particular box type. The instances where this occurred were only with squirrels that shared a testing location (two additional trials for two squirrels) and no squirrels were tested at locations other than their preselected test site.

During the box tests, one of us (C.A.) waited until the squirrel was above ground and near its testing location, after which the appropriate Plexiglas box with the peanut butter was placed at this fixed location at the approximate center of that squirrel's home range. This location was used for all 6 box presentations; therefore, most squirrels had their own test location and received 6 total trials, all at this one location. A video camera (Sony $8 \mathrm{~mm}$ Handycam/CCDFX630) on a 61-cm tripod was placed 1-m from the Plexiglas box. The camera was set to record and the researcher retreated to a vehicle (a distance of 10$57 \mathrm{~m}$; distance did not vary between treatments for any squirrel). If a squirrel did not enter the box before $20 \mathrm{~min}$ had passed, the trial was aborted. For successful trials, a 5-min withdrawal of the animal from the box terminated the trial; therefore, the squirrel's behavior dictated the trial's duration. This trial termination criterion is based on previous observations (e.g. Arenz \& Leger 1997a) that withdrawals of 5 min or greater involve a high frequency of behaviors that are difficult to describe as being related to antipredator vigilance (e.g. grooming, sunning, alternate foraging). Tapes of the trials were viewed on a 48.3-cm Sanyo television with a Panasonic VCR equipped with frame-by-frame viewing capability.

We made a variety of measures either via direct observation during the trial or from the video: (1) total trial duration (from first entry to last withdrawal); (2) mean box entry duration; (3) mean withdrawal duration (4) percentage time withdrawn; (5) and the number, duration, and type of alert postures. Alert postures were categorized as follows: (a) semi-upright alert: on hind feet with a distinctive slouch; (b) upright alert: on hind feet with back straight and mostly perpendicular to the ground; and (c) extended upright alert: same as upright alert except that the squirrel extends its hind legs (Wistrand 1974). The different alert postures occurred outside the box and were differentiated in the analysis by applying a multiplicative factor of 1,2 , or 3 to the measured time spent alert in that posture. A higher level of alertness received a higher factor. In the results, this value is re- 
ferred to as a vigilance score. These multiplicative factors correlate roughly with motivation (i.e. a higher state of alertness) and the height of the eye above the ground (Arenz \& Leger 1997b, 1999). Using this method allowed us to combine the vigilance postures without losing any information regarding the level of alertness, and to determine whether the squirrels' vigilance was affected by the differing visual obstruction patterns produced by the boxes. We did not differentiate between quadrupedal head-up and head-down, because squirrels were usually either feeding within a box, or they were vigilant. Therefore, quadrupedal vigilance is captured in the "percentage of time spent withdrawn" variable.

The planned comparisons which reflect our main hypotheses were C1 vs. O1, $\mathrm{C} 2$ vs. O2, $\mathrm{C} 3$ vs. O3, $\mathrm{O} 1$ vs. O2, $\mathrm{O} 1$ vs. O3, and $\mathrm{O} 2$ vs. O3. These comparisons test for an effect of sky-view obstruction and the effect of increasing sky-view obstruction. If Bonferonni correction is applied to allow for an experiment-wise alpha of 0.05 , each comparison should be evaluated at an alpha of 0.016 , consistent with our directional hypotheses (Keppel \& Zedek 1989). The data were analyzed on SPSS version 5.0.1 and square root transformed to reduce positive skewing. For clarity, the figures display untransformed data.

\section{Results}

Typically, the trials were a sequence of entries and withdrawals into and from the box. The squirrels would approach and enter the box, eat peanut butter, withdraw and visually scan their surroundings, and re-enter the box to feed again. This pattern was repeated until the squirrel withdrew and remained withdrawn for a period of $5 \mathrm{~min}$ or more, terminating the trial.

There were significant differences in the antipredator vigilance of the ground squirrels across the conditions. The squirrels devoted significantly less time and effort to vigilance in the $\mathrm{C} 1$ condition than in any of the three opaque treatments (within-subjects ANOVA: $F_{5,35}=2.33, p=0.063$; two-tailed $t$-tests, $d f=7, p<0.05$ for all planned comparisons). Since the vigilance score does not include quadrupedal vigilance, this is a very conservative test of vigilance differences among the boxes. That is, with this vigilance score, we are only comparing bipedal vigilance postures and perhaps the major difference in antipredator behavior among the boxes is quadrupedal vigilance (see the results for the withdrawal variables below). The squirrels decreased their mean time within the box per entry (within-subjects ANOVA: $F_{5,35}=2.73, p=0.035$ ), but only for the $\mathrm{O} 3$ box (a priori predicted to be the highest risk box) (two tailed $t$-test, $d f=7, p<0.05$ for all comparisons). The pattern in Figure 1(a) is repeated across two other dependent variables, in which the squirrels appear to react differently to boxes with opaque vs. clear eaves. In general, the squirrels spent more time withdrawn per withdrawal (within-subjects ANOVA: $F_{5,35}=2.75, p=0.034$; Figure $\left.1(\mathrm{c})\right)$ and a higher percentage of time withdrawn (within-subjects ANOva: $F_{5,35}=3.93, p=0.006$; Figure $1(\mathrm{~d})$ ) when foraging in the boxes with the opaque eaves vs. boxes with clear eaves.

We found no effect of box type on trial duration (within-subjects ANOvA: $F_{5,35}=2.36, p=0.6$ ), nor were any of the contextual variables significantly differ- 
(a)
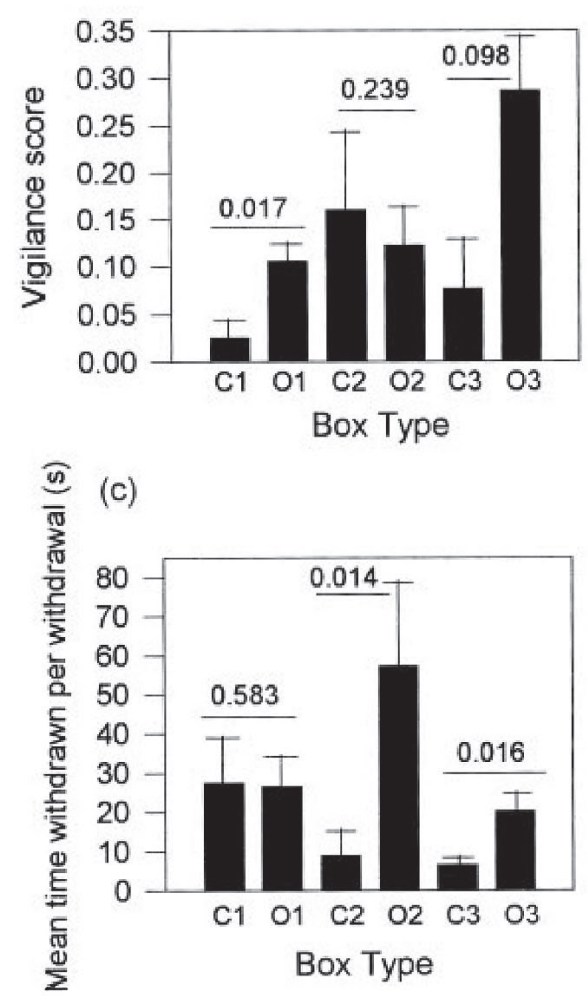

(b)

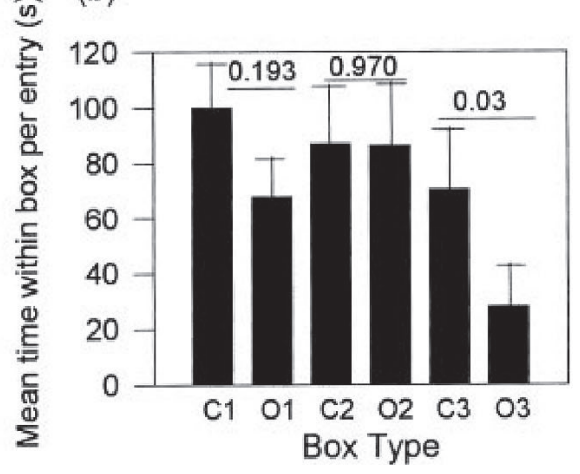

(d)

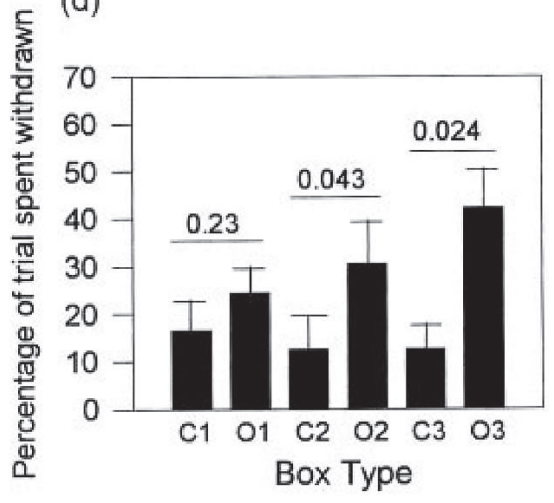

Figure 1. (a) Vigilance score $(\bar{x} \pm S E)$ as a function of box type (b) Time spent within $(\bar{x}$ $\pm S E$ ) each of the boxes per entry (c) Time spent withdrawn ( $\bar{x} \pm$ SE) from box per withdrawal $(d)$ Percentage of trial time $(\bar{x} \pm S E)$ spent withdrawn. Numbers above bars indicate the p-value of the indicated comparison; if Bonferroni correction is applied, the alpha level is 0.016 . See Table 1 for explanation of box type symbols.

ent among the box types (temperature, wind speed, cloud cover; between-subjects ANOVA: all $n^{\prime}$ s). Additionally, there were no significant differences for any measured dependent variable among the three clear box conditions (one-way $t$ tests, all $p>0.05)$.

To allow the reader to compare unmanipulated vigilance values with the vigilance score, we have included Figure 2. Most of the vigilance displayed was semiupright alerts (Figure 2a), resulting in a pattern of vigilance similar to the vigilance score (Figure 2b).

\section{Discussion}

In the present study, we have initiated the process of determining whether ground squirrels are vigilant for aerial predators. Here, through the use of Plexiglas 

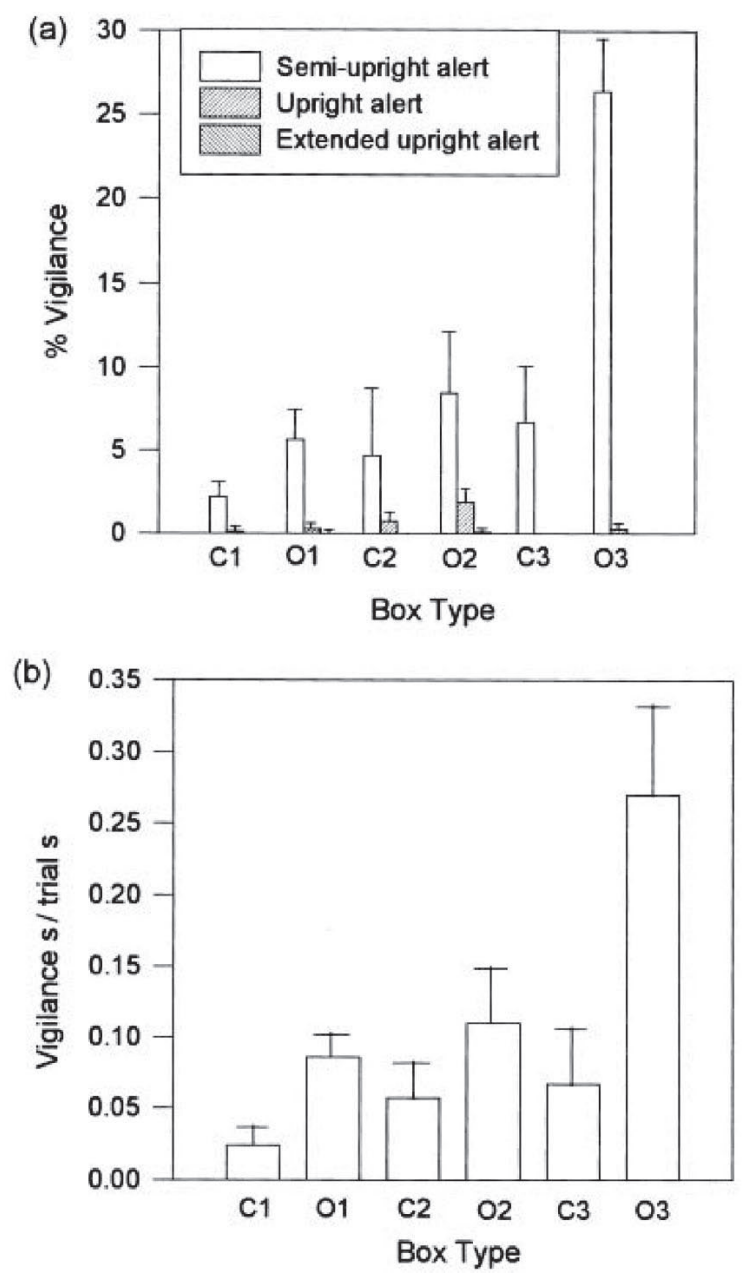

Figure 2. (a) Percentage $(\bar{x} \pm S E)$ of vigilance time spent in each of the three alert postures across box types. (b) The mean ( $\bar{x} \pm$ SE) level of vigilance (vigilance $s /$ trial s) displayed by squirrels for each box type, disregarding alert posture. See Table 1 for explanation of box type symbols.

boxes equipped with rotatable eaves, we have determined the effects of obstructing thirteen-lined ground squirrels' view of the sky. Obstructing a ground squirrel's view of the potential approach of terrestrial predators while allowing it to view the sky (e.g. from horizon to horizon) is logistically difficult. Therefore, we have not yet attempted that experiment. We are not assuming that the ground squirrels can only be vigilant for one or the other classes of predator. However, the hypothesis being tested is that if squirrels react to obstruction of their "sky view" in a manner consistent with the interpretation that it is high risk, then we can conclude that the ground squirrels are vigilant for and monitor the sky for aerial predators. On the other hand, if the ground squirrels had behaved in the same way regardless of clear or opaque eaves, this result would have suggested that the primary focus 
of ground squirrel vigilance is directed at terrestrial predators and/or conspecifics, perhaps as an avoidance of kleptoparasitism. In this study, patterns of effects we might expect that would support the hypothesis that ground squirrel vigilance is directed, in part, at aerial predators are increasing vigilance (a combination of time and posture), increasing the percentage of the trial spent withdrawn, and/or decreasing their mean time within the box per entry.

We found that antipredator vigilance, as measured by percentage of trial time spent withdrawn, and mean time withdrawn per withdrawal were lowest in boxes with clear eaves (Figure 1c,d). This is consistent with the hypothesis that a significant portion of the antipredator vigilance displayed by ground squirrels is directed toward the sky, i.e. above ground level. Therefore, when this portion of the field of view is obstructed, the ground squirrels spend more time withdrawn and are more vigilant than when this visual path is unobstructed. The vigilance score was a composite of three bipedal alert postures and squirrels exhibited a lower score (lower vigilance) in only the $\mathrm{C} 1$ box type. However, since the vigilance score excludes quadrupedal vigilance (included in withdrawal variables, see Methods), it is a highly conservative measure of increased vigilance.

Earlier studies (Arenz \& Leger 1997a, b) found that thirteen-lined ground squirrel adults withdrew significantly more often from boxes that obstructed their lateral view. More specifically, it appears that ground squirrels perceive visual obstruction of their long-range, lateral view as increasing their predation risk. In these former studies, we found that when the squirrels were foraging in higher risk boxes, they reacted by increasing their alertness and the time spent withdrawn, decreasing their mean time within the box per entry, and no significant differences among the various box types in mean time spent withdrawn per withdrawal.

This study adds to our knowledge of the effects of visual obstruction on the perception of predation risk. Although animals make use of their eyes for gathering a variety of information, the evidence provided here indicates that a significant amount of an animal's scanning effort may be directed toward a particular portion of its field of view that is correlated with a high-risk predator class. Our results do not suggest that ground squirrels are ignoring terrestrial threats and future experiments that are designed to determine the relative importance of the two classes of predators (aerial vs. terrestrial) would be very informative.

Acknowledgments - This study was made possible by a NSF Dissertation Improvement Grant (IBN-9701137) and by NSF Grant OSR 9255225. Steffi Jesseau provided valuable field assistance. We thank A. Kamil for his comments on the manuscript and T. J. Valone for his suggestion to use rotatable eaves. We also thank Mike Hutchinson and Wyuka Cemetery for allowing this study to occur on their grounds.

\section{References}

Arenz, C. L. 1997. Handling fox squirrels: Ketamine-hydrochloride versus a simple restraint. Wildl. Soc. Bull., 25, 107-109.

Arenz, C. L. \& Leger, D. W. 1997a. Artificial visual obstruction, antipredator vigilance, and predator detection in the thirteen-lined ground squirrel (Spermophilus tridecemlineatus). Behaviour, 134, 1101-1114. 
Arenz, C. L. \& Leger, D. W. 1997b. The antipredator vigilance of adult and juvenile thirteen-lined ground squirrels (Sciuridae: Spermophilus tridecemlineatus): Visual obstruction and simulated attacks with a hawk model . Ethology, 103, 945-953.

Arenz, C. L. \& Leger, D. W. 1999. Thirteen-lined ground squirrel (Sciuridae: Spermophilus tridecemlineatus) antipredator vigilance decreases as vigilance cost increases. Anim. Behav., 57, 97-103.

Bachman, G. C. 1993. The effect of body condition on the trade-off between vigilance and foraging in Belding's ground squirrels. Anim. Behav., 46, 233-244.

Bekoff, M. 1995. Vigilance, flock size, and flock geometry: information gathering by western evening grosbeaks (Aves, Fringillidae). Ethology., 99, 150-161.

Caine, N. G. \& Marra, S. L. 1988. Vigilance and social organization in two species of primates. Anim. Behav., 36, 897-904.

Dimond, S. \& Lazarus, J. 1974. The problem of vigilance in animal life. Brain. Behav. Evol., 9, 60-79.

Elgar, M. A. 1989. Predator vigilance and group size in mammals and birds: a critical review of the empirical evidence. Biol. Rev., 64, 13-33.

Jones, J. K. Jr, Armstrong, D. M., Choate, J. R. 1985. Guide to the Mammals of the Plains States. University of Nebraska Press, Lincoln.

Keppel, G. \& Zedek, S. 1989. Data Analysis for Research Designs. Analysis of Variance and Multiple Regression/Correlation Approaches. W. H. Freeman, New York.

Lazarus, J. 1990. Looking for trouble. New Scientist, 125, 62-65.

Lazarus, J. \& Symonds, M. 1992. Contrasting effects of protective and obstructive cover on avian vigilance. Anim. Behav., 43, 519-521.

Lima, S. L. 1991. Energy, predators and the behaviour of feeding hummingbirds. Evol. Ecol., 5, 220-230.

Lima, S. L. 1992. Vigilance and foraging substrate: anti-predatory considerations in a nonstandard environment. Behav. Ecol. Sociobiol., 30, 283-289.

Lima, S. L. 1998. Stress and decision making under the risk of predation: Recent developments from behavioral, reproductive, and ecological perspectives. Adv. Study. Behav., 27, 215-290.

Lima, S. L. \& Dill, L. M. 1990. Behavioral decisions made under the risk of predation: A review and prospectus. Can. J. Zool., 68, 619-640.

McCarley, H. 1966. Annual cycle, population dynamics and adaptive behavior of Citellus tridecemlineatus. J. Mamm., 47, 294-316.

Melchior, H. R. \& Iwen, F. A. 1965. Trapping, restraining, and marking Arctic ground squirrels for behavioral observations. J. Wildl. Mgmt., 29, 671-678.

Metcalfe, N. B. 1984. The effects of habitat on the vigilance of shorebirds: Is visibility important? Anim. Behav., 32, 981-985.

Quenette, P.-Y. 1990. Functions of vigilance behavior in mammals: A review. Acta Oecologica, 11, 801-818.

Roberts, G. 1996. Why individual vigilance declines as group size increases. Anim. Behav., 51, 1077-1086.

Sadedin, S. R. \& Elgar, M. A. 1998. The influence of flock size and geometry on the scanning behaviour of spotted turtle doves, Streptopelia chinensis. Austr. J. Ecol., 23, 177-180.

Scheel, D. 1993. Watching for lions in the grass: The usefulness of scanning and its effects during hunts. Anim. Behav., 46, 695-704.

Vestal, B. \& McCarley, H. 1984. Spatial and social relations of kin in thirteen-lined and other ground squirrels. In: The Biology of Ground-Dwelling Squirrels (Murie, J. O., \& Michener, G. R., University of Nebraska Press, Lincoln, pp. 404-423.

Wistrand, H. 1974. Individual, social, and seasonal behavior of the thirteen-lined ground squirrel (Spermophilus tridecemlineatus). J. Mamm., 55, 329-347. 